\title{
Pneumoperitoneum after Endoscopic Duodenal Stent Insertion in a Patient with Percutaneous Transhepatic Biliary Drainage and Biliary Stent: A Case Report
}

\author{
Jinwoo Choi', Min Ji Lee ${ }^{1}$, Hyodeok Lee ${ }^{1}$, Yook Kim², Joung-Ho Han ${ }^{1,3}$ and Seon Mee Park ${ }^{1,3}$ \\ Department of ${ }^{1}$ Internal Medicine, ${ }^{2}$ Radiology, Chungbuk National University Hospital, Cheongju, ${ }^{3}$ Department of Internal Medicine, \\ Chungbuk National University College of Medicine, Cheongju, Korea
}

Early removal of a percutaneous transhepatic biliary drainage (PTBD) tube commonly causes pneumoperitoneum. However, we encountered a patient who developed pneumoperitoneum even with an indwelling PTBD tube. An 84-year-old man was admitted with type III combined duodenal and biliary obstruction secondary to metastatic bladder cancer. A biliary stent was placed using a percutaneous approach, and a duodenal stent was placed endoscopically. A large amount of subphrenic free air was detected after the procedures. Laboratory tests indicated intestinal perforation; however, peritoneal signs were absent. The patient was treated conservatively using an indwelling Levin tube. Seven days later, the massive amount of subphrenic free air disappeared. Follow-up tubography revealed unrestricted bile flow into the small intestine, and the PTBD tube was removed. Prolonged endoscopic procedures in patients with a PTBD tract communicating with the gastrointestinal tract can precipitate pneumoperitoneum. Clinicians should be careful to avoid misdiagnosing this condition as intestinal perforation. Clin Endosc 2019;52:288-292

Key Words: Pneumoperitoneum; Combined duodenal and biliary obstruction; Percutaneous transhepatic biliary drainage; Biliary stenting; Duodenal stenting

\section{INTRODUCTION}

Pneumoperitoneum or pneumomediastinum has been reported in patients undergoing percutaneous biliary stenting or endoscopic retrograde cholangiopancreatography (ERCP) after percutaneous transhepatic biliary drainage (PTBD). ${ }^{1-5}$ Early postprocedural removal of the PTBD tube is the main cause of this complication. ${ }^{6}$ Air is insufflated into the small bowel under pressure during ERCP and leaks into the peritoneal cavity via the unsealed PTBD tract. Thus, a prolonged in-

Received: July 19, 2018 Revised: August 5, 2018

Accepted: August 6, 2018

Correspondence: Seon Mee Park

Department of Internal Medicine, Chungbuk National University College of Medicine, 1 Chungdae-ro, Seowon-gu, Cheongju 28644, Korea

Tel: +82-43-269-6019, Fax: +82-43-273-3252, E-mail: smpark@chungbuk.ac.kr ORCID: https://orcid.org/0000-0002-5835-2741

(c) This is an Open Access article distributed under the terms of the Creative Commons Attribution Non-Commercial License (http://creativecommons.org/ licenses/by-nc/3.0) which permits unrestricted non-commercial use, distribution, and reproduction in any medium, provided the original work is properly cited. dwelling PTBD tube is recommended to prevent this complication. ${ }^{6}$ However, air leakage may occur even in patients with an indwelling PTBD tube. Prolonged endoscopic procedures in patients with an established PTBD tract communicating with the intestinal tract may precipitate pneumoperitoneum, which may be misdiagnosed as intestinal perforation.

We treated a patient who developed pneumoperitoneum after endoscopic duodenal stent insertion, even with a biliary stent and a PTBD tube in place. Abdominal radiography revealed massive leakage of subphrenic air, which usually indicates intestinal perforation. However, in this patient, the air leakage was not caused by duodenal perforation; air had leaked along the PTBD tract through the biliary stent. We report this case with a literature review.

\section{CASE REPORT}

An 84-year-old man was referred to the Gastrointestinal 
Department from the Urology Department with jaundice and poor oral intake. He had been diagnosed with metastatic bladder cancer and had undergone 25 cycles of palliative chemotherapy. However, the cancer had spread to the prostate and multiple lymph nodes. Physical examination upon admission revealed mild epigastric tenderness. Laboratory tests showed a white blood cell (WBC) count of $6,580 \times 10^{9} / \mathrm{L}$; hemoglobin, $10.04 \mathrm{~g} / \mathrm{dL}$; hematocrit, $31.9 \%$; platelet count of $363 \times 10^{12} / \mathrm{L}$; total protein, $7.1 \mathrm{~g} / \mathrm{dL}$; albumin, $3.5 \mathrm{~g} / \mathrm{dL}$; aspartate aminotransferase, $297 \mathrm{U} / \mathrm{L}$; alanine aminotransferase, $205 \mathrm{U} / \mathrm{L}$; alkaline phosphatase, $823 \mathrm{U} / \mathrm{L}$; total bilirubin, $13.17 \mathrm{mg} / \mathrm{dL}$; and C-reactive protein (CRP), $7.44 \mathrm{mg} / \mathrm{dL}$. Abdominal computed tomography (CT) revealed complete obstruction of the distal common bile duct (CBD) and upstream intrahepatic ductal dilatation. Duodenal obstruction and marked dilatation of the stomach and proximal duodenum were also observed. Forward-viewing endoscopy showed a normal papilla and duodenal obstruction below the level of the major papilla. The patient was diagnosed with type III combined duodenal and biliary obstruction (Fig. 1).

ERCP was attempted; however, selective cannulation failed. We immediately switched to the placement of a PTBD tube followed by the insertion of an uncovered self-expandable biliary metal stent measuring $10.5 \mathrm{~mm}$ in diameter and $4 \mathrm{~cm}$ in length (SHSH-08-120-060, Hanarostent; M.I. Tech, Seoul, Korea) through the PTBD, 4 days later (Fig. 2A). The following day, we inserted a bare-metal duodenal stent measuring 24 $\mathrm{mm}$ in diameter and $6 \mathrm{~cm}$ in length (NDSL-18-060-230, Hanarostent; M.I. Tech) into the second and the third portions of the duodenum using forward-viewing endoscopy, under fluoroscopic guidance (Fig. 2B, C). However, follow-up abdominal radiography conducted 2 hours later revealed massive leakage of subphrenic air, and laboratory tests showed a WBC count of $11.8 \times 10^{9} / \mathrm{L}$, CRP $28.27 \mathrm{mg} / \mathrm{dL}$, creatinine $2.20 \mathrm{mg} / \mathrm{dL}$, amylase $310 \mathrm{IU} / \mathrm{L}$, and lipase $476 \mathrm{IU} / \mathrm{L}$. We suspected duodenal perforation based on the laboratory and radiological findings.
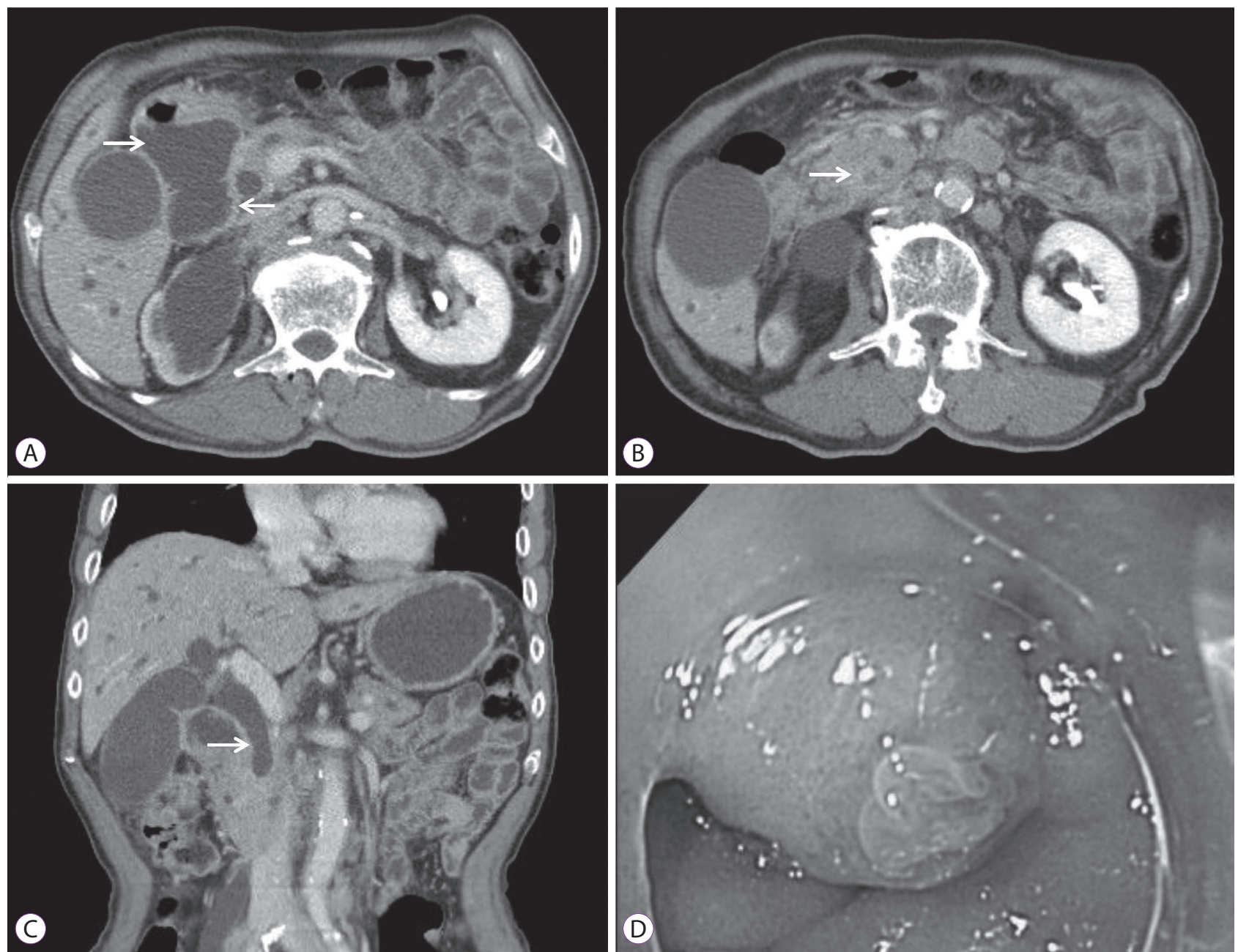

Fig. 1. Abdominal computed tomography and endoscopic images show type III combined duodenal and biliary obstruction. The distended proximal duodenum (A, arrows), luminal stenosis at the distal common bile duct (B, arrow), the proximal bile duct dilatation ( $C$, arrow), and a normal papilla (D) can be observed. 
The patient was immediately placed on an NPO status with the insertion of a Levin tube, and metronidazole was added to his previous antibiotic regimen comprising cefotaxime until culture results were obtained. Abdominal CT showed that the large amount of free intraperitoneal air was not secondary to fluid leakage or an intestinal defect (Fig. 2D). Thereafter, the patient did not complain of any symptoms. His vital signs were within the normal limits and peritoneal signs were absent. Hence, we did not perform an emergency operation and decided to closely observe the patient.

Abdominal radiography conducted three days later showed the persistence of subphrenic air (Fig. 3A), and tubography revealed the delayed passage of bile with incomplete expansion of the duodenal stent (Fig. 3B). Seven days later, the laboratory findings of the patient returned to the normal range, and the massive amount of subphrenic free air disappeared (Fig. 3C, D).
Follow-up tubography revealed unrestricted bile flow into the small intestine, and the PTBD tube was removed. The patient continued to be stable a month later and received hospice care service.

\section{DISCUSSION}

Bile or air leakage into the peritoneal cavity via a PTBD tract manifests as pneumoperitoneum or bile peritonitis. The clinical presentations vary ranging from asymptomatic pneumoperitoneum $^{1-4}$ to peritonitis, ${ }^{5}$ depending on the amount of air or bile leaked through the transhepatic tract. Early removal of a PTBD tube, percutaneous biliary metallic stenting, the rendezvous procedure, and increased air insufflation owing to a prolonged procedure serve as possible contributors. ${ }^{6}$ In
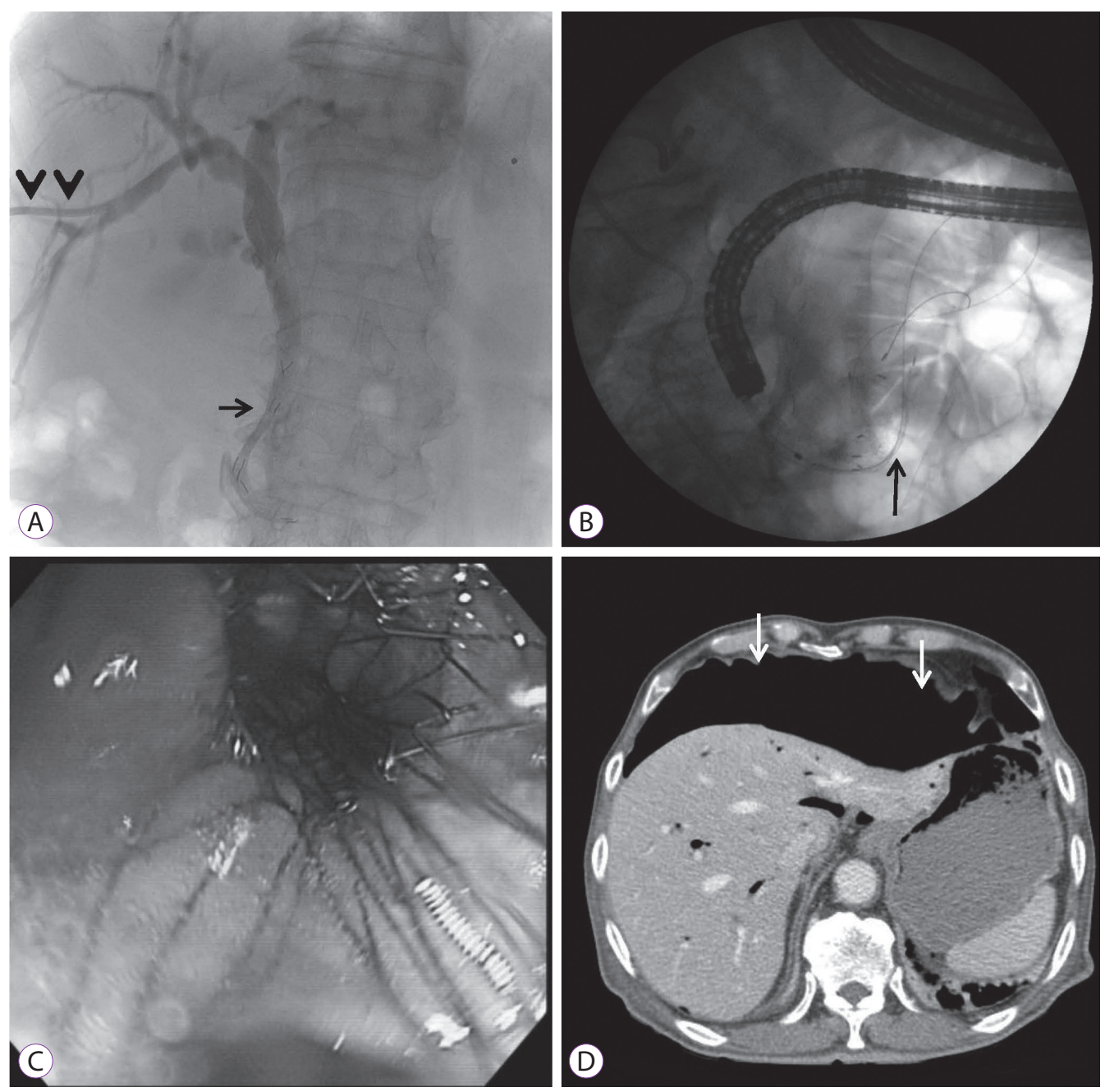

Fig. 2. Images show radiological and endoscopic intervention for type III combined duodenal and biliary obstruction. (A) Biliary metal stent insertion (arrow) through percutaneous transhepatic biliary drainage (arrow heads) and (B) endoscopic duodenal stent insertion after a guidewire has been advanced deep beyond the stricture (arrow). (C) Endoscopic images show incomplete expansion of the duodenal stent. (D) Abdominal computed tomography shows pneumoperitoneum (arrows) after the endoscopic procedure. 

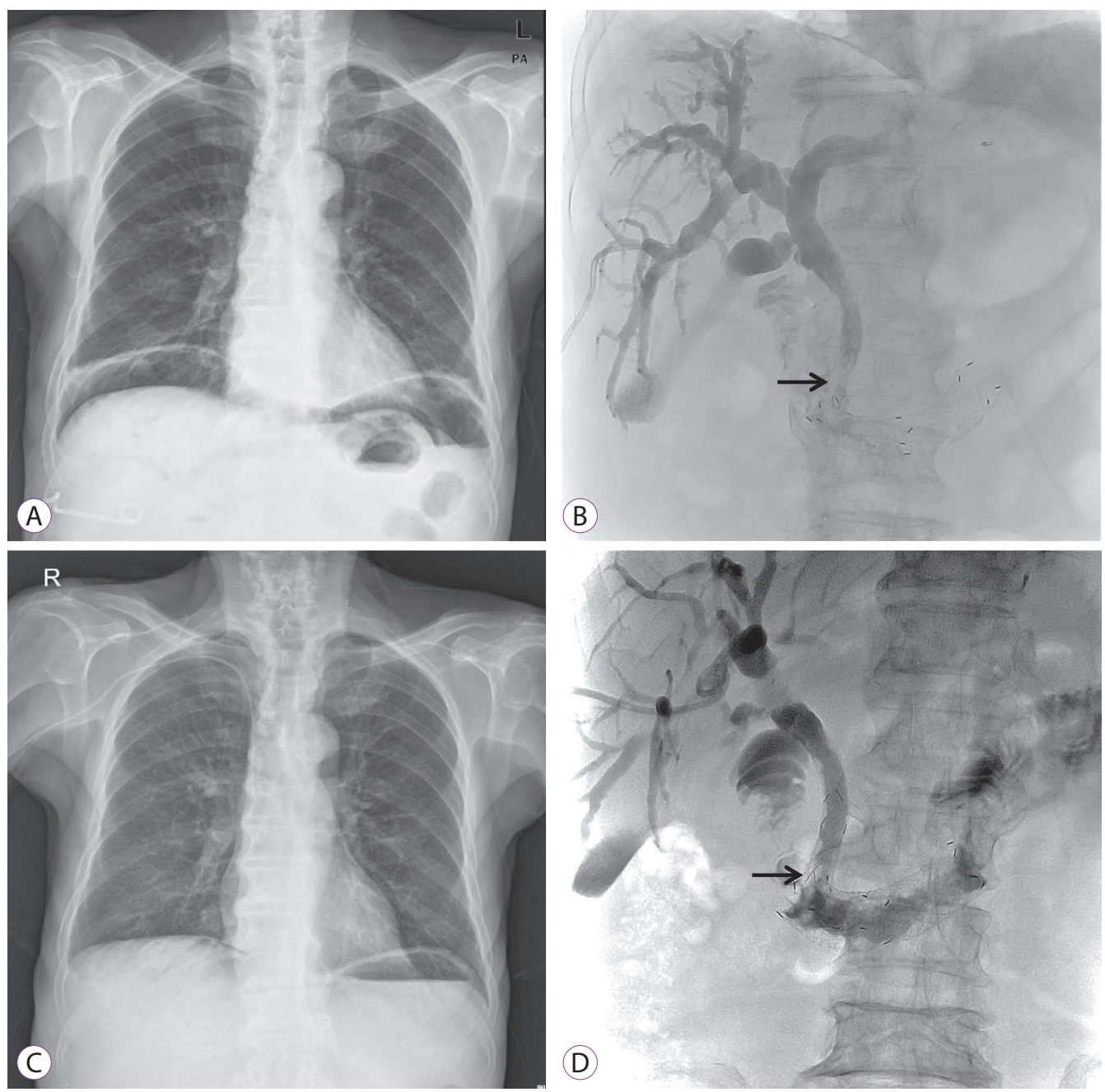

Fig. 3. Abdominal radiography $(A$ and $C)$ and tubography $(B$ and $D)$ images show persistence of subphrenic air $(A)$ with restricted bile flow owing to incomplete expansion of the duodenal stent (B, arrow). Subphrenic air seems to have disappeared $(C)$ following unrestricted drainage of the bile into the intestinal tract after complete expansion of the duodenal stent ( $D$, arrow).

this case, subphrenic free air was unexpectedly observed after duodenal stent insertion in a patient with type III combined duodenal and biliary obstruction. ${ }^{7}$ Although the laboratory findings indicated peritonitis, peritoneal signs were absent. We concluded that the large amount of air and the minimal bile leakage through the PTBD tube were secondary to a prolonged endoscopic procedure, even with an indwelling PTBD tube.

Lee et al. ${ }^{1}$ and Amonkar et al. ${ }^{2}$ have reported pneumoperitoneum and peritonitis caused by transhepatic air and bile leakage after metallic biliary stent placement. Hui et al. ${ }^{3}$ have reported benign pneumoperitoneum (pneumoperitoneum without peritonitis) in patients who underwent a rendezvous procedure with PTBD and endoscopic biliary stenting. Chuang et al. ${ }^{4}$ reported pneumoperitoneum and mild peritonitis after PTBD tube placement and CBD stone removal associated with the rendezvous procedure. The PTBD tube was re- moved at the same time in three cases, ${ }^{1,3,4}$ one day later in one case, $^{2}$ and four days later in one case, ${ }^{2}$ suggesting that early removal of the PTBD tube was a contributory factor. In the current patient, we performed endoscopic procedures with an indwelling PTBD tube and a wide biliary stent placed across the papilla. Despite the indwelling PTBD tube, owing to a prolonged procedure and incomplete expansion of the duodenal stent, the high pressure of the insufflated air caused air to leak through the PTBD tube. In addition, atmospheric air may be sucked into the peritoneum via the percutaneous tract, even with a PTBD tube in place. Interestingly, the subphrenic free air persisted until complete duodenal stent expansion and disappeared after the stent was completely expanded. To prevent this complication, Jonnalagadda et al. ${ }^{6}$ have recommended waiting a few weeks for the PTBD tract to mature before removal of the tube or prior to pursuing endoscopic procedures. However, the optimal timing for safe removal of the PTBD 
tube remains unclear, and delayed endoscopic procedures could be bothersome in these patients.

Previous reports have suggested treatment strategies for PTBD-induced pneumoperitoneum. Postprocedural nasogastric decompression is necessary in patients with abdominal distention until intestinal perforation is excluded. Hui et al. ${ }^{3}$ have suggested the transpapillary injection of N-butyl-2 cyanoacrylate to seal the PTBD tract, which effectively treats enterocutaneous fistulas. ${ }^{8}$ However, its efficacy remains unproven in this condition. In all cases reported in the literature, including the present case, the patients improved within a week with conservative management including the administration of broad-spectrum antibiotics and close observation. Therefore, sealing of the PTBD tract may not be necessary.

We report this case to alert clinicians regarding the possibility of pneumoperitoneum during endoscopic procedures in patients undergoing percutaneous biliary stenting and drainage, and to make them aware that this condition can be managed conservatively.

\section{Conflicts of Interest}

The authors have no financial conflicts of interest.

\section{REFERENCES}

1. Lee JH, Lee DH, Yu JS, Lee SJ, Kwon WC, Kim KW. Pneumoperitoneum caused by transhepatic air leak after metallic biliary stent placement. Cardiovasc Intervent Radiol 2000;23:482-484

2. Amonkar SJ, Laasch HU, Valle JW. Pneumoperitoneum following percutaneous biliary intervention: not necessarily a cause for alarm. Cardiovasc Intervent Radiol 2008;31:439-443.

3. Hui YT, Lam WM, Lam TW, Cheung WC, Sze SF, Wong CT. Benign pneumoperitoneum developed after endoscopic biliary metallic stent placement with the rendezvous procedure. Gastrointest Endosc 2008;67:179-180.

4. Chuang $\mathrm{CH}$, Chen CY, Tsai HM. Pneumoperitoneum caused by air leakage through the percutaneous puncture tract as a complication of rendezvous technique: a case report. Kaohsiung J Med Sci 2008;24:614617.

5. Virgilio E, Chieco PA, Salaj A, et al. Conservative management of pneumoperitonitis after percutaneous transhepatic insertion of metallic biliary stents. Am Surg 2015;81:E418-E419.

6. Jonnalagadda S, Azar R. Avoiding pneumomediastinum during ERCP after percutaneous transhepatic biliary drainage. Gastrointest Endosc 2008;68:809; author reply 809-810.

7. Baron TH. Management of simultaneous biliary and duodenal obstruction: the endoscopic perspective. Gut Liver 2010;4 Suppl 1:S50-S56.

8. Lisle DA, Hunter JC, Pollard CW, Borrowdale RC. Percutaneous gelfoam embolization of chronic enterocutaneous fistulas: report of three cases. Dis Colon Rectum 2007;50:251-256. 